\title{
His Bundle Pacing but not Left Bundle Pacing Corrects Septal Flash in Left Bundle Branch Block Patients
}

\author{
Marina Strocchi ${ }^{1}$, Aurel Neic ${ }^{2}$, Matthias A F Gsell ${ }^{4}$, Christoph M Augustin ${ }^{4}$, Julien Bouyssier ${ }^{3}$, \\ Karli Gillette $^{4}$, Mark K Elliot ${ }^{1}$, Justin S Gould ${ }^{1}$, Jonathan M Behar ${ }^{1}$, Baldeep Sidhu ${ }^{1}$, Martin J \\ Bishop $^{1}$, Edward J Vigmond ${ }^{3}$, Gernot Plank ${ }^{4}$, Christopher A Rinaldi ${ }^{1}$, Steven A Niederer ${ }^{1}$ \\ ${ }^{1}$ King's College London, London, UK \\ ${ }^{2}$ NumeriCor GmbH, Graz, Austria \\ ${ }^{3}$ IHU Liryc, Fondation Bordeaux Université, Pessac, France \\ ${ }^{4}$ Medical University of Graz, Graz, Austria
}

\begin{abstract}
His bundle pacing $(H B P)$ and left bundle pacing $(L B P)$ are novel delivery methods for cardiac resynchronisation therapy (CRT) for left bundle branch block (LBBB) patients. Septal flash $(S F)$, an abnormal pre-ejection motion of the septum towards the left ventricle $(L V)$ arising from dyssynchronous activation, has been shown in the past to be a robust and independent predictor for CRT response. Although small-cohort studies showed the feasibility and efficacy of HBP and LBP, the effects of HBP and LBP on septal motion have yet to be investigated. In this study, we used our four-chamber heart electro-mechanics simulation framework to determine whether $H B P$ and $L B P$ can correct for $S F$. We performed simulations in four fourchamber heart models. In synchronous and LBBB activation, simulated mean lateral septal movement from the right ventricle $(R V)$ into the $L V$ was $-0.4 \pm 0.5 \mathrm{~mm}$ and $3.7 \pm 0.9 \mathrm{~mm}(p<0.05)$, respectively. HBP reduced septal motion to $-0.4 \pm 0.5 \mathrm{~mm}$ ( $p=0.5$ when compared to synchronous activation). In LBP, septal motion was reversed to $0.9 \pm 0.5 \mathrm{~mm}$ and significantly different from synchronous activation $(p<0.05)$. HBP was better able to recover septal function over LBP in patients with complete atrioventricular block.
\end{abstract}

\section{Introduction}

Cardiac resynchronisation therapy (CRT) is one of the most effective treatments for left bundle branch block (LBBB) patients. However, still 30-40\% of patients do not respond to standard CRT[1], delivered through one apical RV lead and one epicardial LV lead. Recently, His bundle pacing (HBP) and left bundle pacing (LBP) have been emerging as novel delivery methods for CRT, as pacing the high conduction His-Purkinje system instead of the slow-

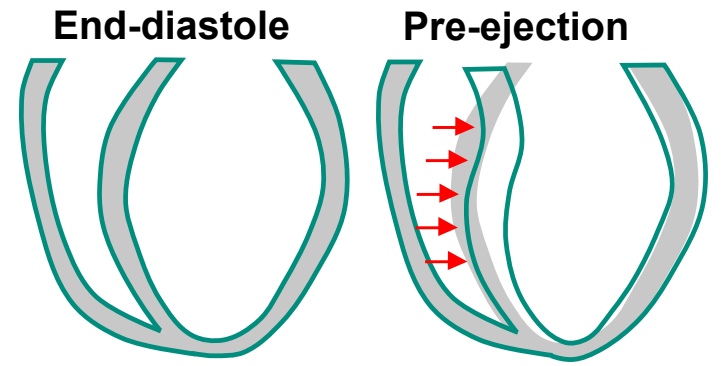

Figure 1. Septal flash. At pre-ejection, early RV activation causes the septum to move towards the $\mathrm{LV}$, which activates late due to LBBB and is therefore still quiescent.

conducting myocardium might restore native synchronous activation and lead to better ventricular function. Although small-cohort and single patient studies showed the feasibility and efficacy of both HBP and LBP in restoring electrical synchrony of the heart[2], there is still a lot to be understood about their effects on heart function. Furthermore, there are indications of negative effects of LBP on the right ventricle (RV) that have yet to be investigated.

Response to CRT has been correlated in the past with correction of septal flash (SF) [3], an abnormal pre-ejection motion of the septum towards the left ventricle (LV), Figure 1. Most commonly assessed through visual inspection by echocardiography, and strain analysis based on speckle tracking, SF has been identified as a direct consequence of ventricular dyssynchrony caused by LBBB. SF has been shown to be a robust and independent predictor for CRT response, with patients diagnosed with SF having better long-term survival[3]. Although correction of SF after CRT was reported to have good sensitivity and specificity for predicting long-term outcome[3], the effects of HBP and LBP on septal motion have not been investigated.

In this study, we applied our electro-mechanics simula- 
tion framework to four heart failure patient to quantify the effects of HBP and LBP on septal motion.

\section{Methods}

\subsection{Model generation}

We segmented four-chamber heart tetrahedral meshes inclusive of ventricular myofiber orientation from ECGgated CT images acquired from four heart failure patients with a pipeline described previously[4] (Figure 22. The meshes used in this study are available for download on Zenodo (https://doi.org/10.5281/ zenodo.3890034).
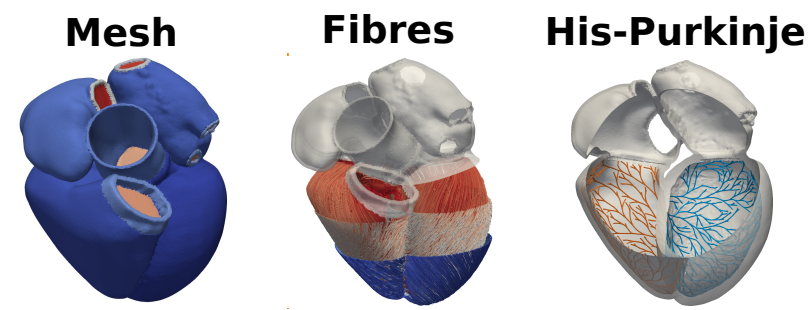

Figure 2. Model generation. The images show a fourchamber heart mesh (left), corresponding ventricular fibre orientation (center) and generated His-Purkinje network (right).

A His-Purkinje system was generated on the LV and RV endocardial surfaces of each mesh using an open source code (https://github.com/fsahli/ fractal-tree), Figure 2. The Purkinje system was coupled with ventricular myocardium by connecting each terminal node of the Purkinje with the surrounding myocardium nodes to allow for stimulus propagation from the His-Purkinje network to the myocardium and viceversa[2]. We simulated LBBB by cutting the connection of the LV Purkinje system with the rest of the network along the His bundle.

\subsection{Electro-mechanics simulation}

We simulated electrical activation of the ventricles with a reaction-eikonal model[5]. Ventricular myocardium was modelled as a transversely isotropic medium with the preferred direction of propagation aligned with the local myofibre orientation. Ventricular myocardium conduction velocity $(\mathrm{CV})$ was set to $0.6 \mathrm{~m} / \mathrm{s}$ and $0.24 \mathrm{~m} / \mathrm{s}$ in the fibre and transverse direction[2], respectively. CV of the HisPurkinje tree was set to $4.0 \mathrm{~m} / \mathrm{s}[2]$.

The activation times computed on the ventricles were used to trigger active tension rise in the ventricular myocardium, simulated with a phenomenological model[6],

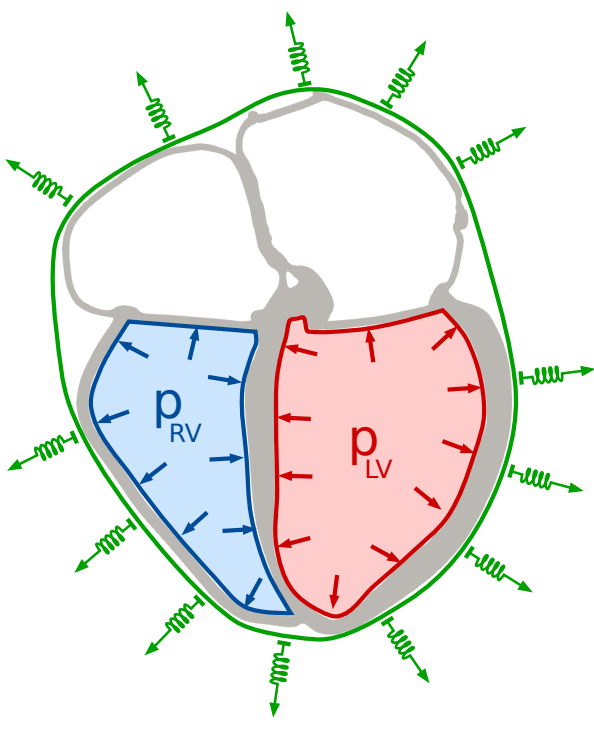

Figure 3. Mechanics boundary conditions. We constrained the outer surface of the heart with normal springs to represent the effect of the pericardium on the heart (green). The LV (red) and RV (blue) endocardial surfaces were applied with pressure adapted to match the outflow computed with two three-element Windkessel models.

discarding the effect of length-dependence. Peak in active tension, twitch duration, rising time and decay time were set to $135 \mathrm{kPa}, 550 \mathrm{~ms}, 130 \mathrm{~ms}$ and $100 \mathrm{~ms}$, respectively[4]. The motion of the heart was constrained with normal springs on the outer surface of the heart to represent the effect of the pericardium (Figure 3, green) [4,7]. Motion tracking applied to ECG-gated CT images acquired on the cohort showed that the roof of the atria and the regions close to the apex of the ventricles moved the least[7]. Therefore, we scaled normal spring stiffness to constrain these regions the most. Regions around the base of the ventricles were not constrained to allow for apico-basal displacement during ventricular systole.

LV and RV pressure-volume relationships were simulated as described previously[7, 8]. LV preload was represented with a constant pressure of $14.6 \mathrm{mmHg}$ based on invasive pressure data available from the patient cohort. Since RV pressure data were not available, RV enddiastolic pressure was computed by scaling the LV pressure. Ventricular afterload was represented with two threeelement Windkessel models. LV afterload parameters were set based on the literature[9]. Parameters for RV afterload were scaled from LV parameters[6]. Passive ventricular mechanics was represented with the Guccione model, while non-ventricular tissue was represented with a Neohookean model. Parameters were based on the literature [4.7]. We applied this electro-mechanics framework on four heart failure patients, using the same parameters and 
boundary conditions for all meshes.

We simulated LBBB baseline conditions for all four patients first. Then, we simulated selective HBP and LBP by pacing the His bundle below the block and the left bundle as it emerged on the LV endocardium. For comparison, we also ran a simulation with synchronous activation (no LBBB). We computed mean septal displacement normal to the surface during ventricular systole, with negative and positive displacements representing septal motion towards the $\mathrm{LV}$ and the RV, respectively.

\section{Results}

\section{1. $\quad$ LBBB baseline simulation}

Figure 4 shows results for LBBB baseline simulations for all patients. Our simulated activation reproduced typical features of LBBB activation, with early RV and late LV free wall activation (Figure 4A). Mean simulated LV and RV latest activation time (LAT) were $140 \pm 9 \mathrm{~ms}$ and $106 \pm 13 \mathrm{~ms}$, respectively. Figure $4 \mathrm{~B}$ shows changes in average septal displacement projected normal to the surface over time, from LV end-diastole to LV end-systole. Early RV systole led to SF in all patients, with an increased pre-ejection peak in mean septal motion of $-3.7 \pm 0.9 \mathrm{~mm}$ (Figure $4 \mathrm{~B}$, circles) compared to $-0.4 \pm 0.5 \mathrm{~mm}$ during synchronous activation.

\subsection{Septal motion in HBP and LBP}

HBP resulted in resynchronised ventricular activation. LV LAT during HBP was reduced compared to baseline (HBP: $107 \pm 6 \mathrm{~ms}$ vs LBBB: $140 \pm 9 \mathrm{~ms}$ ), while RV LAT remained unchanged (HBP: $106 \pm 11 \mathrm{~ms}$ vs LBBB: $107 \pm 11 \mathrm{~ms})$. Resynchronised ventricular activation resulted in correction of SF. Figure 5A shows septal displacement during ventricular systole at baseline (solid line) and during HBP (dotted line) for one patient as a representative example. The negative peak in septal motion at ventricular pre-ejection disappeared, Figure $5 \mathrm{~A}$, blue arrow (HBP: $-0.4 \pm 0.5 \mathrm{~mm}$ vs LBBB: $-3.7 \pm 0.9 \mathrm{~mm}$ ). HBP led to similar septal motion simulated with synchronous activation (synchronous: $-0.4 \pm 0.5 \mathrm{~mm}$ ), indicating correction of SF.

Similarly to HBP, LBP reduced LV LAT (LBP: $107 \pm 5 \mathrm{~ms}$ vs LBBB: $140 \pm 9 \mathrm{~ms}$ ) from baseline, indicating more synchronous LV activation. However, as opposed to HBP which did not effect RV activation, LBP caused delayed RV activation (RV LAT LBP: $131 \pm 8 \mathrm{~ms}$ vs LBBB: $106 \pm 11 \mathrm{~ms})$. During LBP, only the LV is paced and the activation wave can travel to the RV only once the LV is activated, causing the RV to be activated late. In the model, this delay translated into an abnormal septal motion towards the RV (LBP: $0.9 \pm 0.5 \mathrm{~mm}$, Figure $5 \mathrm{~B}$, blue
A
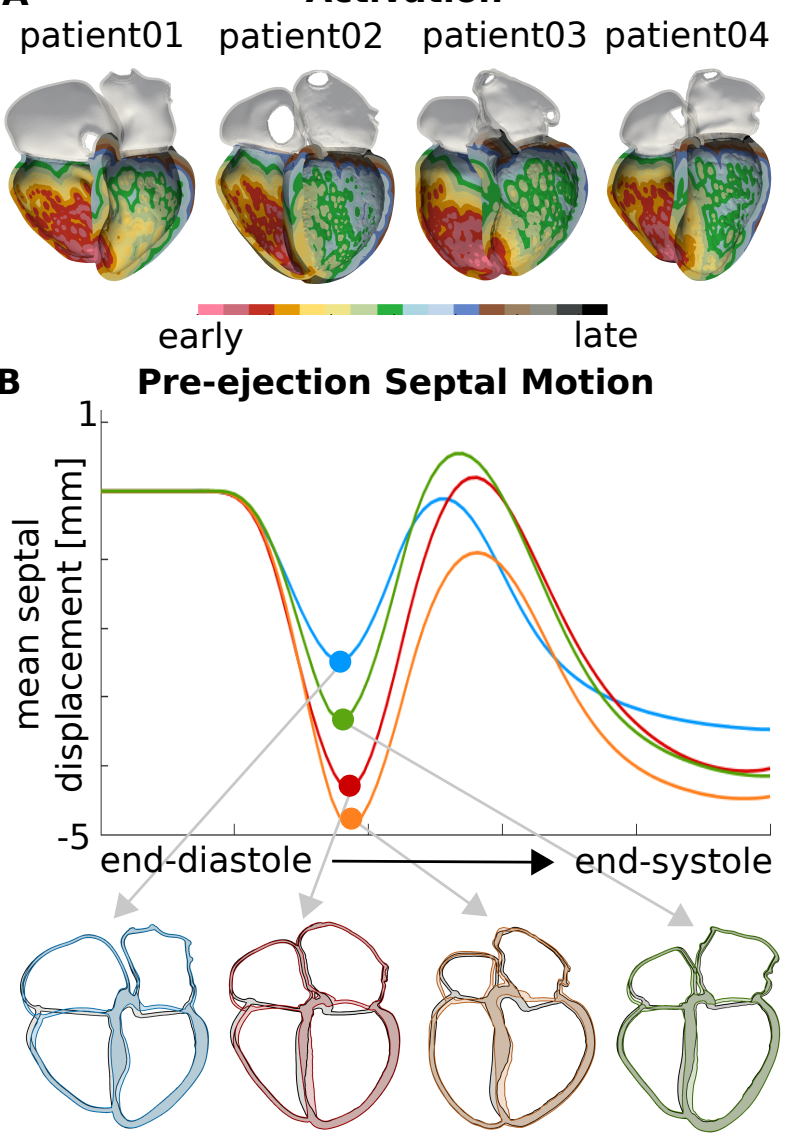

Figure 4. Baseline results. A Activation. The top row shows the LBBB baseline activation for all four patients. B Septal motion. The plot shows the average displacement of the septum normal to the surface from end-diastole to end-systole, with negative displacement representing septal motion towards the LV. Each line represents one patient. The bottom row shows the corresponding motion of the four-chamber heart at peak of negative septal displacement at pre-ejection (SF) for all four patients compared to end-diastole (gray).

arrow) because the RV is quiescent while the LV starts contracting, as opposed to HBP and synchronous activation $(-0.4 \pm 0.5 \mathrm{~mm})$.

\section{Conclusions}

While HBP resynchronises ventricular activation without affecting RV activation, resulting in restored normal septal motion, LBP leads to slow RV activation, causing abnormal rightwards septal motion. Baseline SF might be used in clinical practice to predict which patients are more likely to respond to HBP. More studies are needed in order to investigate the effect of LBP on RV function. 

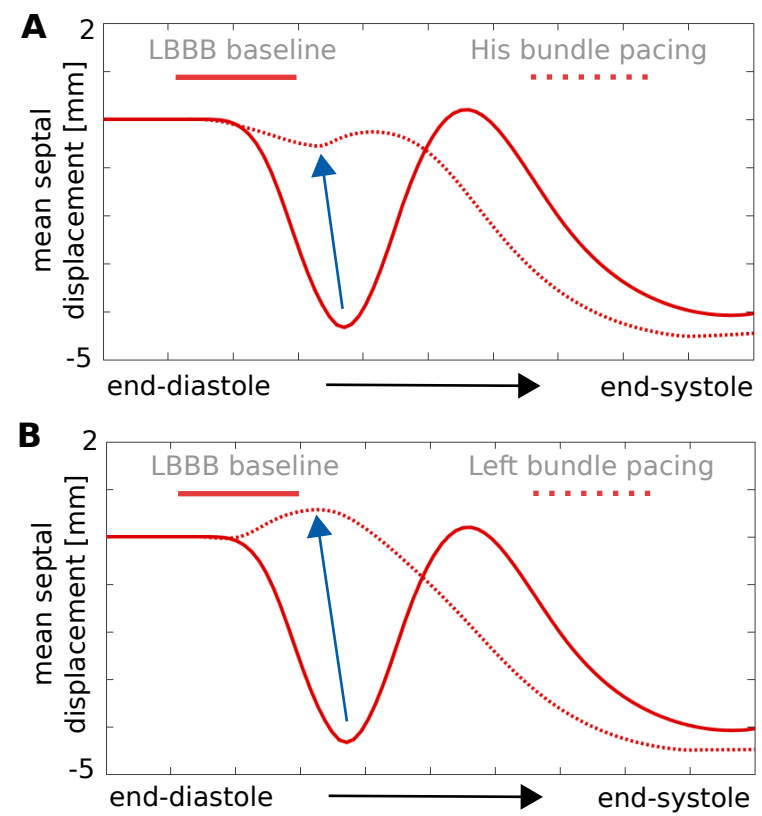

Figure 5. Septal motion - HBP. The plot shows the average displacement of the septum normal to the surface from end-diastole to end-systole for one patient as a representative example. Negative displacement represents septal motion towards the LV. A LBBB (solid line) vs HBP (dotted line). B LBBB (solid line) vs LBP (dotted line).

\section{Acknowledgements}

This study received support from the UK Engineering and Physical Sciences Research Council (EP/M012492/1, NS/A000049/1, EP/L015226/1 and EP/P01268X/1), the Wellcome EPSRC Centre for Medical Engineering (NS/A000049/1 and WT 203148/Z/16/Z), the British Heart Foundation (PG/15/91/31812 and PG/13/37/30280), and Kings Health Partners London National Institute for Health Research (NIHR) Biomedical Research Centre. Dr Vigmond E.J., Dr Bouyssier J. and Dr Plank G. were supported by the ERA-Net ERACoSysMed Co-Fund 2015 (ANR 15 CMED 0003 01) and the Austrian Science Fund (FWF) (PI2760-B30). Dr Vigmond E.J. and Dr Bouyssier J. were supported by the Investments of the Future program managed by the National Research Agency (ANR), Grant reference ANR-10-IAHU-04. Dr Augustin C. M. was supported by the Marie Skłodowska-Curie fellowship (GA No 750835) by the European Unions Horizon 2020 research and innovation program. Miss Strocchi M. was supported by an unrestricted Abbott educational grant through the Centre for Doctoral Training in Medical Imaging at Kings College London. This work made use of ARCHER, the UKs national high-performance computing service located at the University of Edinburgh and funded by the Office of Science and Technology through Engineering and Physi- cal Sciences Research Councils High End Computing Programme.

\section{References}

[1] Thomas G, Kim J, Lerman BB. Improving cardiac resynchronisation therapy. Arrhythmia Electrophysiology Review 2019;8(3):220.

[2] Strocchi M, Lee AW, Neic A, Bouyssier J, Gillette K, Plank G, Elliott MK, Gould J, Behar JM, Sidhu B, et al. His bundle and left bundle pacing with optimised atrio-ventricular delay achieve superior electrical synchrony over endocardial and epicardial pacing in left bundle branch block patients. Heart rhythm 2020;.

[3] Stankovic I, Prinz C, Ciarka A, Daraban AM, Kotrc M, Aarones M, Szulik M, Winter S, Belmans A, Neskovic AN, et al. Relationship of visually assessed apical rocking and septal flash to response and long-term survival following cardiac resynchronization therapy (predict-crt). European Heart Journal Cardiovascular Imaging 2016;17(3):262-269.

[4] Strocchi M, Augustin CM, Gsell MA, Karabelas E, Neic A, Gillette K, Razeghi O, Prassl AJ, Vigmond EJ, Behar $\mathrm{JM}$, et al. A publicly available virtual cohort of fourchamber heart meshes for cardiac electro-mechanics simulations. PloS one 2020;15(6):e0235145.

[5] Neic A, Campos FO, Prassl AJ, Niederer SA, Bishop MJ, Vigmond EJ, Plank G. Efficient computation of electrograms and ecgs in human whole heart simulations using a reaction-eikonal model. Journal of computational physics 2017;346:191-211.

[6] Niederer SA, Plank G, Chinchapatnam P, Ginks M, Lamata P, Rhode KS, Rinaldi CA, Razavi R, Smith NP. Lengthdependent tension in the failing heart and the efficacy of cardiac resynchronization therapy. Cardiovasc Res oct 2010; 89(2):336-343.

[7] Strocchi M, Gsell MA, Augustin CM, Razeghi O, Roney CH, Prassl AJ, Vigmond EJ, Behar JM, Gould JS, Rinaldi $\mathrm{CA}$, et al. Simulating ventricular systolic motion in a fourchamber heart model with spatially varying robin boundary conditions to model the effect of the pericardium. Journal of Biomechanics 2020;101:109645.

[8] Augustin CM, Crozier A, Neic A, Prassl AJ, Karabelas E, Ferreira da Silva T, Fernandes JF, Campos F, Kuehne T, Plank G. Patient-specific modeling of left ventricular electromechanics as a driver for haemodynamic analysis. EP Europace 2016;18(suppl_4):iv121-iv129.

[9] Fritz T, Wieners C, Seemann G, Steen H, Dössel O. Simulation of the contraction of the ventricles in a human heart model including atria and pericardium. Biomech Model Mechanobiol 2014;13(3):627-641.

\section{Address for correspondence:}

Marina Strocchi

King's College London

St Thomas' Hospital, Westminster Bridge Road

SE1 7EH, London, UK

marina.strocchi@kcl.ac.uk 